Revue d'histoire de l'Amérique française

Q4. REVUE D'HISTOIRE DE L'AMÉRIQUE FRANÇAISE

\title{
Le difficile pari de la reconstruction du parti conservateur fédéral au Québec (1925-1926)
}

\section{Nelson Michaud}

Volume 52, numéro 1, été 1998

URI : https://id.erudit.org/iderudit/005550ar

DOI : https://doi.org/10.7202/005550ar

Aller au sommaire du numéro

Éditeur(s)

Institut d'histoire de l'Amérique française

ISSN

0035-2357 (imprimé)

1492-1383 (numérique)

Découvrir la revue

Citer cet article

Michaud, N. (1998). Le difficile pari de la reconstruction du parti conservateur fédéral au Québec (1925-1926). Revue d'histoire de l'Amérique française, 52(1), 23-46. https://doi.org/10.7202/005550ar
Résumé de l'article

Été 1926. La vie politique canadienne est en crise puisque ses institutions démocratiques paraissent menacées. Pour la première fois, il semble impossible de constituer un gouvernement stable. Au centre de cette crise, on trouve deux partis, deux hommes: les libéraux de William Lyon Mackenzie King et les conservateurs d'Arthur Meighen se disputent le droit de diriger le pays. Pour les conservateurs, la commande est de taille puisqu'ils ressentent encore profondément, surtout au Québec, les retombées de leur politique conscriptionniste. Cet article analyse les stratégies utilisées par l'équipe de Meighen pour reconquérir la confiance d'un électorat sceptique, mais non sans avoir d'abord relevé les défis que présentent un parti divisé, un pays déchiré et un Parlement ambivalent.
Tous droits réservés @ Institut d'histoire de l'Amérique française, 1998
Ce document est protégé par la loi sur le droit d'auteur. L'utilisation des services d'Érudit (y compris la reproduction) est assujettie à sa politique d'utilisation que vous pouvez consulter en ligne.

https://apropos.erudit.org/fr/usagers/politique-dutilisation/ 


\title{
LE DIFFICILE PARI DE LA RECONSTRUCTION DU PARTI CONSERVATEUR FÉDÉRAL AU QUÉBEC (1925-1926)
}

\author{
NELSON MICHAUD \\ Département de science politique \\ Université Dalhousie
}

\begin{abstract}
RÉSUMÉ
Été 1926. La vie politique canadienne est en crise puisque ses institutions démocratiques paraissent menacées. Pour la première fois, il semble impossible de constituer un gouvernement stable. Au centre de cette crise, on trouve deux partis, deux hommes: les libéraux de William Lyon Mackenzie King et les conservateurs d'Arthur Meighen se disputent le droit de diriger le pays. Pour les conservateurs, la commande est de taille puisqu'ils ressentent encore profondément, surtout au Québec, les retombées de leur politique conscriptionniste. Cet article analyse les stratégies utilisées par l'équipe de Meighen pour reconquérir la confiance d'un électorat sceptique, mais non sans avoir d'abord relevé les défis que présentent un parti divisé, un pays déchiré et un Parlement ambivalent.
\end{abstract}

\section{ABSTRACT}

Summer 1926. Commotion and agitation characterise the Canadian political life; a major crisis threatens its democratic institutions. For the first time, it seems impossible to constitute a viable government. Two men, two parties are the key players at the heart of the crisis: William Lyon Mackenzie King's liberals and Arthur Meighen's conservatives. For the latter, the task is heavy since they carry the weight of their conscriptionist policy, a burden which is particularly present in Québec. This paper presents an analysis of the strategies used by Meighen's team to conquer a skeptical electorate, but not without having first taken up the challenges presented by a divided party, a torn country, and an ambivalent Parliament.

Les élections fédérales canadiennes de l'été de 1926 visaient avant tout à mettre un terme à l'une des périodes d'incertitude politique les plus sévères qu'a connues le pays. À toutes fins utiles, le scrutin précédent, tenu en octobre 1925, n'avait pu déterminer de vainqueur: les conservateurs remportent la pluralité des sièges (116/245), mais sont à court de sept voix pour s'assurer d'une majorité en Chambre. Comble de malheur pour le parti d'Arthur Meighen, le Québec n'a élu, sous la 
bannière conservatrice, que 4 de ses 65 députés et tous 4 proviennent de circonscriptions à forte majorité anglophone. Le Premier ministre sortant, le libéral William Lyon Mackenzie King, convainc le gouverneur général Byng de Vimy que, malgré la victoire conservatrice, c'est lui qui est davantage en mesure de former un gouvernement: il dit pouvoir compter sur l'appui des tiers partis ${ }^{1}$, notamment du Parti progressiste dont 22 des 24 députés viennent des provinces des Prairies. Or, dans un tel contexte, l'instabilité s'installe vite et on anticipe que King sera bientôt chassé du pouvoir.

De fait, dès juin 1926, soit moins de six mois après que le Parlement eut été convoqué, King fait face à un vote de confiance que son gouvernement ne semble plus être en mesure de remporter. Plutôt que d'affronter l'adversité, King demande au gouverneur général de dissoudre le Parlement et d'appeler une élection générale. Le refus du vice-roi d'accéder à la demande du Premier ministre provoque un imbroglio constitutionnel notoire, à la suite duquel un nouveau Premier ministre est désigné par de Vimy: il s'agit du chef conservateur, Arthur Meighen. Le climat politique dans lequel Meighen accepte le mandat ne laisse planer aucun doute: en cet été de 1926, le Canada est devenu un pays ingouvernable et il faudra vraisemblablement un nouveau scrutin pour briser l'embâcle politique qui gêne la gouverne du pays. En ce sens, non seulement Meighen cherche-t-il à asseoir la direction qu'il entend donner au pays sur un mandat populaire plus solide mais, pour ce faire, il doit se réconcilier avec le Québec - et particulièrement avec le Québec francophone - qui avait tourné le dos aux tories de Robert Borden, à propos de la question des écoles ontariennes ${ }^{2}$ et, surtout, à cause de leur politique conscriptionniste et de la mise en place du gouvernement d'Union en $1917^{3}$. Cette rupture avait été confirmée notamment par les résultats de l'élection précédente.

Certes, Meighen s'était depuis longtemps efforcé de jeter des ponts vers le Québec en vue d'une telle élection: il avait approché un exministre conservateur fédéral, démissionnaire de 1917, réélu à la Législature provinciale en 1923 et figure en vue du parti au Québec: Ésioff-

1. Ces tiers partis sont les progressistes (24 sièges) et les travaillistes ( 2 sièges). Deux candidats indépendants et deux libéraux indépendants ont aussi été élus.

2. Voir à ce sujet Nelson Michaud, «Les écoles d'Ontario ou le dilemme des conservateurs québécois: confrontation des principes nationalistes et de la réalité politique», Revue d'histoire de l'Amérique française, 49,3 (hiver 1996): 395-417.

3. Voir Réal Bélanger, L'impossible défi. Albert Sévigny et les conservateurs fédéraux (1902-1918) (Sainte-Foy, Les Presses de l'Université Laval, coll. «Cahiers d'histoire», nº 27, 1983), $368 \mathrm{p}$. 
Léon Patenaude 4 . Patenaude avait bien accepté de faire le saut dans l'arène fédérale en 1925 mais, à la grande déception du chef tory, il avait poursuivi une campagne indépendante de celle de l'équipe conservatrice canadienne. Â la lumière des résultats serrés de 1925, Meighen réévalue non seulement la stratégie envers son lieutenant québécois, mais aussi celle que les événements le pressent d'adopter en vue d'un scrutin anticipé.

Patenaude fera la campagne de 1926 et il la fera, cette fois, aux côtés de Meighen. Comment ce retour sera-t-il orchestré? En fonction de quoi Patenaude pourra-t-il se présenter, de façon plausible et crédible, comme le lieutenant de Meighen, alors que les deux hommes avaient adopté des positions antagonistes lors du débat entourant le service militaire obligatoire en 1917 et, surtout, alors que Patenaude s'est déclaré «aussi indépendant de M. Meighen que [...] de M. King» lors de l'élection de 1925? Nos recherches, particulièrement celles menées dans le fonds inédit de Patenaude, nous amènent à considérer que les politiciens tentent d'utiliser la recette éprouvée en 1911 des valeurs nationalistes canadiennes, notamment celles de la reconnaissance de l'autonomie canadienne à l'intérieur du giron impérial britannique et de la dualité ethnique de la nation canadienne. Le tout s'est cependant révélé un exercice bancal.

Mais si l'on s'intéresse aux hommes, c'est aussi pour comprendre un parti en butte à des difficultés majeures. L'historiographie nous a peu appris quant à cette difficile reconstruction que tente alors le Parti conservateur fédéral. Nous proposons donc d'analyser ce pari difficile dans lequel s'engage ce parti qui cherche à redevenir un parti vraiment national et non seulement une organisation politique basée en Ontario, comme le laissaient croire les derniers résultats électoraux. Pour ce faire, il semble que Meighen ait mis l'accent sur la reconquête politique du Québec. La solution à tous les maux ne pourrait être aussi simple. Les difficultés que rencontre le parti sont de quatre niveaux: le parti en tant que tel, la nature dichotomique du Canada d'alors, le Parlement ambivalent dans ses choix et un électorat difficile à convaincre en pareilles circonstances. En fait, c'est parce que le Parti conservateur n'a pas su résoudre l'ensemble de ces difficultés de façon satisfaisante qu'il ne réussit pas à prendre le pouvoir lors des élections de 1926. Il ouvrait du coup la porte sur l'ère quasi ininterrompue de pouvoir libéral qui, à quelques mandats près, devait durer près de soixante ans.

4. Le chapitre de Nelson Michaud, «Le nationalisme tel que senti par les Québécois: une nécessité pour Arthur Meighen?» dans l'ouvrage de Michel Sarra-Bournet, Les nationalismes au Québec, $X I X^{e}$ et XX $X^{e}$ siècles (Montréal, Éditions Québec/Amérique, 1998) donne un bon aperçu de ces tentatives. 


\section{LES DIFFICULTÉS D'UN PARTI FACE À LUI-MÊME LES PREMIÈRES ÉTAPES}

La dynamique politique a ses exigences, d'autant plus lorsque le contexte ravit aux joueurs un atout précieux: le temps. Cela est particulièrement vrai en cette fin d'année 1925. Les stratèges conservateurs savent que, pour conquérir l'électorat, il faudra davantage qu'une machine électorale unifiée: il s'agit d'effacer les «errements» de la campagne électorale qui s'est achevée à peine deux mois plus tôt: la manœuvre de réconciliation devra projeter une image d'unité qui sera importante puisqu'il faudra notamment effacer le différend entre les supporters de Patenaude et ceux qui ne lui pardonnent pas d'avoir écarté le chef national Meighen.

Ainsi, la lutte pour la direction du Parti conservateur au Québec dans laquelle se sont engagés Patenaude et Rodolphe Monty ${ }^{5}$ refait surface. Cette scission est aussi notoire lorsqu'on remarque qu'un Armand Lavergne, seul autre candidat meigheniste lors du scrutin de 1925, s'abstient de participer aux réunions de relance du parti ${ }^{6}$ organisées par Patenaude dès décembre 1925 et janvier 1926 ${ }^{7}$. Le pari de la réunification des forces conservatrices s'annonce vraiment difficile à tenir. Pour y arriver, Patenaude lance des appels au respect de la doctrine conservatrice, à l'appui au chef et à la mise sur pied d'une organisation solide afin de restaurer l'unité du parti ${ }^{8}$.

Moins d'une semaine après la première rencontre, les actions alors dévolues au comité d'organisation sont davantage concentrées entre les mains d'un organisateur en chef qui devra ramener son parti dans le cœur de l'électorat québécois francophone. À la suite de discussions, Meighen et Patenaude choisissent ${ }^{9}$ le sénateur David-Ovide L'Espérance qui cède

5. Appelé au Cabinet par Meighen en 1920, Monty était, aux élections de 1925, l'un des deux supporteurs meighenistes du Québec, mais n'avait pas su pour autant gagner la confiance de Meighen. Voir Archives nationales du Canada (ANC), Fonds Lavergne, Meighen à Lavergne, 24 décembre 1925; Fonds Meighen, Patenaude à Meighen, 4 avril 1926; Archives privées de l'Industrielle-Alliance (APA), Fonds Patenaude, Boîte II, «Chemise Politique - Élections 1926», Meighen à Patenaude, 7 avril 1926.

6. ANC, Fonds Meighen, Lavergne à Meighen, 28 décembre 1925.

7. APA, Fonds Patenaude, Boîte II, Chemise «Campagne 1925 dans Bagot», «Caucus conservateur du 22 décembre 1925 à Hôtel Place Viger», 29 p. Ce procès-verbal est le seul élément nous permettant de connaître le contenu des délibérations puisque la presse n'y va que d'hypothèses et de suppositions d'après les propos glanés ça et là, la séance de travail se déroulant à huis clos. Voir aussi idem, Chemise «Politique - Campagne 1926», «Procès-verbal de la réunion conservatrice tenue au Château Frontenac de Québec, le 9 janvier 1926», n.p.

8. Idem.

9. La candidature du sénateur semble relever d'un consensus auquel sont parvenus Meighen et Patenaude. 
aux pressions et accepte d'orchestrer l'organisation du parti au Québec ${ }^{10}$. Ce choix n'est toutefois pas facile: les oppositions à la candidature de l'ancien conservateur-nationaliste de 1911 sont fortes.

Plusieurs groupes de politiciens conservateurs semblent en effet s'opposer au choix du sénateur. D'abord, il y a ses frères d'armes de 1911, ceux qui appartenaient au groupe des conservateurs-nationalistes, groupe duquel L'Espérance s'est rapidement dissocié pour finalement accepter un siège au Sénat en 1917. Parmi eux, il faut d'abord compter Albert Sévigny ${ }^{11}$. On compte aussi Pierre-Édouard Blondin qui reçoit même une lettre de Meighen le pressant de ne pas brouiller les négociations entreprises avec L'Espérance ${ }^{12}$. Mais celui qui offre la plus vive opposition reste sans conteste Armand Lavergne. Fort de ses racines politiques dans le comté de Montmagny, terre d'attache du sénateur, Lavergne connaît L'Espérance de longue date et ce n'est pas d'hier qu'il ne le porte pas en haute estime ${ }^{13}$. Dès 1924, flairant sans doute l'ascendance que L'Espérance peut avoir sur son chef, Lavergne ne ménage pas les lettres et enjoint Meighen de ne pas s'associer au sénateur; ce faisant, il ne laisse planer aucun doute sur ses sentiments ${ }^{14}$. Malgré les invitations répétées du chef à tendre vers l'unité et à collaborer avec L'Espérance ${ }^{15}$, Lavergne ne peut s'y résoudre. Dix-huit mois après ses premières recommandations, il n'a toujours pas changé d'idée ${ }^{16}$.

Pis encore, la contestation de L'Espérance est aussi soutenue par certains des principaux organisateurs du parti, tels J. C. Lockwell, Antoine Rivard, Lucien Moraud et J. S. O'Meara qui n'ont guère prisé que le nouvel organisateur en chef les ait ignorés lorsqu'il a convoqué une réunion

10. ANC, Fonds Meighen, Meighen à L'Espérance, 15 janvier 1926 et L'Espérance à Meighen, 14 janvier 1926. Si la réponse arrive avant la demande, c'est que Meighen avait d'abord approché verbalement L'Espérance, la lettre officialisant la demande devant suivre. Voir aussi, idem, Meighen à Patenaude, 28 décembre 1925. De même que APA, Fonds Patenaude, Boîte II, Chemise «Campagne 1926», Lucien Moraud à Patenaude, 25 janvier 1926. L'Espérance lui aurait confié que «malgré les sacrifices considérables qu'il avait faits pour le parti, M. Meighen l'avait forcé de faire ce nouveau sacrifice et c'est après des refus réitérés qu'il avait finalement consenti à accepter».

11. ANC, Fonds Borden, Journal personnel, 7 avril 1916.

12. ANC, Fonds Meighen, Meighen à Blondin, 28 décembre 1925; Patenaude à Meighen, 28 décembre 1926 [sic]. Il s'agit d'une erreur de datation: la lettre est de 1925 et non de 1926 puisqu'il y est question de l'entrée prochaine en fonction de L'Espérance comme organisateur, ce qui survient en 1926.

13. Marc Laterreur l'illustre bien dans son opuscule Armand Lavergne (Montréal, Fides, 1968), 55-62

14. ANC, Fonds Meighen, Lavergne à Meighen, 28 mai 1924, 11 juin 1924.

15. ANC, Fonds Lavergne, Meighen à Lavergne, 12 décembre 1925. Déjà, le chef avait aussi tenté de démontrer que les qualités de L'Espérance pouvaient en faire un homme utile au parti. Voir à ce sujet, ANC, Fonds Meighen, Meighen à Lavergne, 30 mai 1924.

16. ANC, Fonds Lavergne, Lavergne à Arthur Lalonde, 21 juin 1926. 
d'organisation du district de Québec ${ }^{17}$. Stratégie d'organisateur afin d'évaluer les ressources moins connues du parti? Provocation visant à connaître à quel point les politiques tiennent vraiment à faire partie du projet? Les deux scénarios sont possibles. Mais le geste du sénateur L'Espérance, pour hautement tactique qu'il soit, ne fut pas très apprécié par les organisateurs locaux.

Cette zizanie quasi orchestrée constitue un obstacle de plus à franchir. Patenaude n'a d'autre choix que de restaurer l'unité du Parti en vue des élections que l'on sent prochaines: il opte alors pour la diplomatie et tente de réconcilier les factions. Il retient tout d'abord Lockwell qui avait décidé d'abandonner la barque ${ }^{18}$, puis il contient la fureur d'un Lucien Moraud tout aussi irrité par la situation. Ses efforts portent fruit, car un autre disciple, O'Meara, lui rapportera que les deux hommes semblent accepter la décision «de Meighen», même sans enthousiasme ${ }^{19}$. L'harmonie n'est pas restaurée pour autant, car s'il est vrai que Moraud et Lockwell acceptent de se ranger, ils n'en poursuivent pas moins leurs revendications auprès de Patenaude ${ }^{20}$ qu'ils considèrent comme «le seul autorisé officiellement par les candidats et les organisateurs de la province pour régler cette situation ${ }^{21} \gg$.

Ce contexte, on ne peut plus délicat, suffit presque, à lui seul, à expliquer le comportement tout en nuance qu'affiche Patenaude face aux revendications de ses hommes et aux désirs de son chef, avec qui il semble tout à fait en accord. Patenaude laisse se dérouler les événements, il laisse agir le temps. À ceux qui lui demandent conseil, le «maître du silence» suggère qu'«il serait sage d'attendre un peu avant d'intervenir ${ }^{22} \gg$.

En agissant ainsi, Patenaude reçoit de solides appuis, dont celui du sénateur Lorne $\mathrm{C}$. Webster, homme de confiance de Meighen en matière d'organisation. Celui-ci convainc d'ailleurs son chef, impatient de voir l'unité du parti rétablie, qu'il s'agit là de la meilleure route à suivre ${ }^{23}$. L'évaluation de Webster s'avère juste car, dès la fin d'avril, Joseph Morin, député provincial et bras droit de Patenaude, informe Meighen que ce

17. APA, Fonds Patenaude, Boîte II, Chemise «Politique - Campagne 1926», Lucien Moraud à Patenaude, 25 janvier 1926.

18. Idem, Lockwell à Patenaude, 21 janvier 1926.

19. Idem, O'Meara à Patenaude, 25 janvier 1926.

20. Idem, Moraud à Patenaude, 23 février 1926; Lockwell à Patenaude, 2 mars 1926

21. Idem, Du même au même, 2 mars 1926. Le fait que le comité d'organisation n'ait pas eu voix au chapitre lors du choix de l'organisateur en chef constitue l'un des griefs du groupe de Québec.

22. Idem, Patenaude à Louis Normand, 25 février 1926. Patenaude tiendra d'ailleurs un discours semblable à l'intention de Lockwell. Idem, Patenaude à Lockwell, 3 mars 1926.

23. ANC, Fonds Meighen, Webster à Meighen, 22 janvier 1926. 
dernier a su rallier le chef provincial Arthur Sauvé, de même que le comité d'organisation ${ }^{24}$. Au cours de l'été, L'Espérance témoignera luimême à Meighen ${ }^{25}$ de la bonne collaboration qui existe entre Patenaude et lui.

Patenaude aura donc atteint un premier objectif, celui d'amorcer la réconciliation du parti sur des bases solides. Si la position qu'il a adoptée y a contribué, il ne faudrait pas pour autant sous-estimer le travail de L'Espérance. Pour établir sa crédibilité, ce dernier effectue une tournée de reconnaissance dans la province qui lui confirme ce que J.-S. Royer avait décrit à Meighen: «Il y a tant de factions dans le parti qu'il est nécessaire d'avoir le support de toutes ${ }^{26} . \gg$ En politicien d'expérience, L'Espérance décide d'ériger son édifice politique sur les points communs des diverses factions plutôt que de chercher à bâtir sur des fissures à demi colmatées. Ce terrain d'entente semble celui de la doctrine, celui des «valeurs nationales» que Patenaude s'acharne à garder dans son discours. Dans cette optique, l'étape cruciale du plan de réorganisation consiste en l'acquisition d'un quotidien, outil de prédilection pour propager le message. Cette réalisation répond, de plus, au souhait de plusieurs et permet de cimenter les liens entre les hommes de Patenaude et ceux de L'Espérance. C'est ainsi que le 19 juillet 1926, La Patrie devient officiellement l'organe francophone du Parti conservateur fédéral au Québec, ce qui est confirmé à la une de l'édition du lendemain ${ }^{27}$.

La phase de reconstruction ainsi couronnée, L'Espérance peut maintenant travailler à la réconciliation afin de tirer Patenaude des coulisses pour le ramener à l'avant-scène, aux côtés de son chef. Cette accolade publique que se donneront Patenaude et Meighen chapeaute une démarche entreprise par le chef tory immédiatement après les élections de 1925. Pour bien en saisir le sens, il est toutefois nécessaire d'en suivre le cheminement complet, un exercice qui nous demande d'intercaler ici un retour en arrière et qui nous fournira des éléments analytiques utiles pour replacer cette gigantesque entreprise de reconstruction dans le contexte des difficultés politiques que le Canada de 1925-1926 rencontre.

\footnotetext{
24. APA, Fonds Patenaude, loc. cit., Morin à Meighen, avril 1926.

25. ANC, Fonds Meighen, L'Espérance à Meighen, 14 juillet 1926.

26. APA, Fonds Patenaude, loc. cit., Royer à Meighen, 21 janvier 1926. Le problème est majeur: non seulement l'unification a-t-elle été la préoccupation principale des réunions patenaudistes de décembre et de janvier, mais plus de $75 \%$ des lettres de cette section du fonds Patenaude font état d'extrêmes morcellements qui existent au sein de l'aile québécoise du Parti conservateur fédéral. 27. La Patrie, «Ce que sera La Patrie», 20 juillet 1926, 1.
} 


\section{LES DIFFICULTÉS D'UN PARTI NATIONAL FACE À UN PAYS DIVISÉ}

L'ardent désir que Meighen caresse de revoir Patenaude à ses côtés ${ }^{28}$, la disponibilité de celui-ci ${ }^{29}$, de même que la communion de pensée des deux politiciens sur l'avenir et l'organisation du parti ${ }^{30}$, voilà autant de facteurs qui favorisent le rapprochement. En fait, Meighen est prêt à beaucoup pour que ses efforts soient couronnés de succès puisque, comme le souligne James Murray Beck, «since Meighen considered King's early defeat [in Parliament] to be inevitable he [...] thought only in terms of the next election ${ }^{31} \gg$.

Il est important de rappeler que nous sommes pratiquement aux lendemains de la Guerre et de la crise de la conscription qui, à peine huit ans plus tôt, sévissait. Les Canadiens français y avaient vu une mesure pour les forcer à participer à une guerre qu'ils ne considéraient pas être la leur, un reniement des promesses faites par les conservateurs - dont Patenaude - lors de la mise sur pied du Service national ${ }^{32}$. Malgré un sentiment autonomiste grandissant, qui aurait pu le rapprocher des sentiments politiques véhiculés au Québec, le Canada anglais gardait une certaine rancœur vis-à-vis de ses compatriotes francophones qui ne s'émouvaient pas outre mesure lorsque les fanfares entonnaient le Rule Britannia. Ce ressentiment était d'autant plus amer que, comme le souligne David William Paterson, les Ontariens, notamment, avaient vu leur perception de la guerre passer d'une candeur patriotique enthousiaste pour les grandeurs et les besoins de l'Empire, à une réserve morose lorsque furent affichées les premières listes des hommes tombés au champ d'honneur ${ }^{33}$. La perte qui d'un fils, qui d'un frère, qui d'un époux, alliée aux rumeurs d'une

\footnotetext{
28. ANC, Fonds Meighen, Meighen à R. C. Matthews, 5 avril 1926.

29. Idem, Blondin à Meighen, 24 décembre 1925. Voir aussi Idem, J.-S. Royer à Meighen, 21 janvier 1926.

30. On connaît déjà les vues de Patenaude à ce sujet. Pour Meighen, la question de la réorganisation du parti et son avenir notamment au Québec, «has been an increasing worry to [him]», ANC, Fonds Meighen, Meighen à Webster, 19 mars 1926. Le chef conservateur va même jusqu'à affirmer que «The situation has troubled me away great deal and the condition which confronts me is exceedingly difficult. I earnestly ask you to cooperate to the utmost to enable a solution to be found.», APA, Fonds Patenaude, Meighen à Joseph Morin, 2 avril 1926.

31. James Murray Beck, Pendulum of Power. Canada's Federal Elections (Scarborough, Prentice Hall, 1968), 177.

32. Le Service national consistait en une vaste campagne visant à faire l'inventaire des ressources dont disposait le Canada. Présidé par le député de Calgary, R. B. Bennett, l'exercice était toutefois perçu par plusieurs, et particulièrement au Québec, comme le prélude à l'enrôlement obligatoire, perception contre laquelle Patenaude et ses collègues avaient lutté.

33. David William Paterson, Loyalty, Ontario and the First World War, mémoire de maîtrise, Université McGill, 1986, 82, 102.
} 
participation mitigée de l'élément francophone du pays, ne pouvaient qu'exacerber les passions.

C'est dans ce contexte, habilement saisi par Thompson et Seager ${ }^{34}$, que Meighen tente de reconquérir l'électorat francophone du Québec, sans s'aliéner le Canada anglais. On imagine facilement que l'entreprise n'est pas aisée: face au pays qu'il veut gouverner, Meighen doit tenir un discours qui plaise aux deux clientèles, tout en restant sincère et crédible. Meighen entend donc se rapprocher du Québec, d'abord par le discours, puis par sa présence, avant de tendre officiellement la main à Patenaude.

Élément qui peut paraître incongru, Patenaude n'est présent à aucune de ces étapes. L'incongruité s'explique toutefois mieux si l'on considère qu'après s'être déclaré indépendant de son chef, Patenaude ne pouvait revenir publiquement auprès de Meighen dès le lendemain du cuisant échec de 1925. Ce faisant, il aurait eu l'air du politicien opportuniste et aurait, du coup, affaibli son autorité au Québec, autorité dont il avait grandement besoin pour reconstruire le parti. De son côté, Meighen ne pouvait davantage donner l'impression de céder facilement aux pressions de Patenaude, sans faire preuve d'un manque de leadership et sans donner au Canada anglais l'impression d'abdiquer devant le Québec. Pour chacun d'eux et pour le parti, le prix politique à payer aurait été trop élevé. Cela n'empêchera toutefois pas les deux hommes de continuer leurs pourparlers en coulisse. C'est ainsi qu'il fut convenu que Meighen devait, petit à petit, officiellement se rapprocher du Québec, puis de son lieutenant. Cela ne se fera toutefois pas sans heurter les sentiments de certains milieux canadiens-anglais.

Cette démarche étapiste se concrétise dès octobre 1925 à Hamilton, lors d'une allocution de Meighen. Il y définit sa position quant aux relations que le Canada doit entretenir avec l'Empire britannique, notamment sur le plan militaire. Sa position vise une plus grande autonomie canadienne et rejoint du coup les préoccupations défendues par Patenaude depuis 1910. Dans cette déclaration, le chef conservateur affirme que désormais, avant de s'engager dans un conflit impérial ou avant d'adopter une attitude neutre, le gouvernement canadien devra tenir une élection référendaire sur la question. En principe, voilà de quoi bien amorcer le rapprochement souhaité. Patenaude reçoit en effet les déclarations de Meighen avec une évidente satisfaction, qu'il lui exprime clairement: «Les déclarations que vous avez faites ont été accueillies avec beaucoup

34. John Herd Thompson et Allan Seager, Canada 1922-1939: Decades of Discord (Toronto, McClelland and Stewart, 1985). 
de faveur, écrit-il. C'est un grand pas dans la bonne voie et j'espère que vous ne vous arrêterez pas en si bon chemin. Je vous félicite ${ }^{35}$.»

L'accueil réservé aux propos du chef tory n'est toutefois pas unanimement favorable. Pour les impérialistes et les orangistes ontariens, ce discours est anathème et constitue une flagrante contradiction avec le fameux «Ready! Aye! Ready! We stand by you!» qu'il avait lancé lors de la crise du Chanak ${ }^{36}$, assurant alors l'Empire de la participation canadienne ${ }^{37}$. L'Ontario et l'Ouest le font rapidement sentir au principal intéressé, notamment par la voix de leurs journaux. Par ailleurs, chez les autonomistes de l'envergure d'Henri Bourassa, on pourrait croire à une satisfaction certaine mais, au contraire, le député de Labelle considère l'intervention de Meighen comme pure stupidité: advenant l'éclatement d'un conflit entre la Grande-Bretagne et la France, la consultation populaire qui serait menée au Canada dégénérerait bien vite en guerre civile, prédit-il ${ }^{38}$. L'effet escompté par Patenaude et Meighen n'est donc pas celui obtenu. Comme le déplore Meighen lui-même, «there has been a lot of misunderstanding about the Hamilton speech ${ }^{39}{ } \gg$. En fait, confie-t-il à Armand Lavergne, «these difficulties have been much greater than I had anticipated ${ }^{40} \gg$.

35. ANC, Fonds Meighen, Patenaude à Meighen, 24 novembre 1925.

36. À la suite du traité de Versailles, l'Empire ottoman est partagé entre la France, l'Italie et l'Angleterre. Mustafa Kémal, leader politique turc, essaie de récupérer les terres cédées aux alliés. Il règle avec les gouvernements français et italien, mais ne peut en arriver à une entente avec l'Angleterre qui défend Constantinople (Istanbul). Le conflit armé est imminent et la région de Chanac en serait le théêtre. Face à la situation, le Premier ministre britannique, le travailliste James Ramsay Macdonald, demande aux Dominions s'ils sont prêts à participer à l'effort de guerre. Les journaux s'emparent de la nouvelle et font dire à Macdonald qu'il avait demandé l'aide directe des membres de l'Empire. Nuance subtile, mais fort importante. En réponse à la dépêche, le Premier ministre canadien King s'insurge: le Canada ne participera pas à une guerre avant que le Parlement ne soit saisi de la question. Les Chambres étant dissoutes au moment où l'incident se produit, King espère qu'une solution au conflit sera trouvée avant que les Communes ne soient saisies de la question. Il agit en politicien gambler. Il joue... et gagne: une entente entre Kémal et Macdonald est conclue avant la convocation du Parlement canadien. C'est en réponse à King que Meighen avait lancé son fameux «Ready! Aye! Ready!». Voir C. P. Stacey, Canada and the Age of Conflict, II: 1921-1948, the Mackenzie King Era (Toronto, University of Toronto Press, 1981), 17-27 et T. Glazebrook, A History of Canadian External Relations, II: In the Empire and the World, 1914-39 (Toronto, McClelland \& Stewart, 1966), 63-67, édition révisée. Cet incident, en apparence anodin, marque tout de même un tournant politique dans l'évolution du nationalisme canadien, car même la population anglo-saxonne du pays a favorablement accueilli la prise de position de King.

37. Meighen se défend toutefois d'être l'instigateur de cette position, prétendant que l'appel aurait d'abord été lancé par sir Wilfrid Laurier. Voir ANC, Fonds Meighen, Meighen au docteur R. Morand, député, 21 janvier 1926.

38. Le Devoir, «Si M. Meighen a changé», 7 septembre 1926, 3.

39. ANC, Fonds Meighen, Meighen au sénateur R.-H. Pope, 16 janvier 1926.

40. ANC, Fonds Lavergne, Meighen à Lavergne, 12 décembre 1925. 
Meighen ne se laisse pas rebuter pour autant. Il ne regrette rien et se dit prêt à confondre ses détracteurs ${ }^{41}$. D'autant plus que l'accueil d'ÉsioffLéon Patenaude à son discours est porteur d'espoir, sentiment auquel il s'agrippe. Meighen est, en effet, plus que jamais décidé d'aller chercher le Québec, cet as qui, croit-il, lui permettra d'abattre, lors du prochain scrutin, une main gagnante. La mort du député fédéral libéral de Bagot $^{42}$ devait l'aider en ce sens. Dans le contexte politique de cet automne de 1925, l'élection complémentaire prend une importance extraordinaire: une victoire conservatrice signifierait bien davantage qu'un député de plus dans le camp de Meighen; elle serait vraisemblablement interprétée comme le rejet de l'équipe King qui s'accroche tant bien que mal au pouvoir. Tout le peuple du Canada, de l'Atlantique au Pacifique, a hâte de connaître l'issue du scrutin. Au soir du verdict, les libéraux conservent le siège.

Les conservateurs font tout de même des gains appréciables au décompte officiel. Mais c'est surtout au point de vue stratégique qu'ils gagnent de précieux galons: cette élection offre l'occasion de combler davantage le fossé officiel qui existe toujours, en théorie, entre Meighen et Patenaude. Même s'il semble que l'on évalue comme précipité le retour de Patenaude lui-même, le chef conservateur, lui, vient prendre part à la lutte. Meighen en profite pour redorer son image et celle de son parti, visitant écoles et presbytères et s'adressant aux foules en français, ce qui lui vaut un accueil que l'on dit chaleureux ${ }^{43}$. Le résultat semble d'autant plus encourageant que le contenu du message lance un pont vers le Québec et vers Patenaude.

Ainsi, lorsque, dans un discours à Acton Vale, Meighen définit sa vision de la nation canadienne, ses propos s'alignent sur les convictions de Patenaude ${ }^{44}$. D'abord, le chef conservateur réitère en français ses propos de Hamilton. Puis, concernant les relations impériales, les domaines étatique et territorial, la place des «deux $\operatorname{races}^{45}$ » ou l'économie, Meighen

41. Ibid.

42. J.-E. Marcile avait été élu sur son lit de mort, atteint d'un cancer en phase terminale. Il avait pu compter sur «la pitié de nos gens pour leur vieux bonhomme de député». ANC, Fonds Meighen, G.-A. Roy à Meighen, 2 novembre 1925.

43. ANC, Fonds Meighen, renferme plusieurs lettres en ce sens dont celles d'André Fauteux (10 novembre 1925), L.-J. Gauthier (19 novembre 1925), S. F. Tolmie (28 novembre 1925), etc. Souvent, elles font état de sentiments partagés par d'autres politiciens, tels les Lorne C. Webster, Thomas Chapais et autres, de même que par certains groupes venant surtout du comté. Les invitations faites au chef tory se firent nombreuses.

44. L'Événement, «M. Meighen royalement reçu à Bagot», 2 décembre 1925, 7.

45. Il faut ici comprendre le concept de «races» selon le sens qu'on lui donne à l'époque, soit celui de «peuples». Les deux races auxquelles Meighen fait ici allusion sont les peuples francophone et anglophone, les «deux peuples fondateurs» du Canada. 
reprend le discours de son lieutenant convoité. Et, comme si l'appel à Patenaude n'était pas assez clair, Meighen ajoute: «Nous suivons la politique de Macdonald et Cartier ${ }^{46}$, leitmotiv tout patenaudiste.

Après les déclarations de Hamilton, la communion de pensée de Bagot, il ne reste plus qu'à célébrer la réconciliation totale et définitive entre les deux politiciens. Pourquoi, alors, faut-il attendre plus de six mois pour voir la chose se concrétiser? Souvenons-nous que l'élection dans Bagot a lieu au début de décembre 1925. Il faut laisser le temps à Patenaude de regrouper ses hommes et de canaliser les forces du parti vers un objectif commun. Voilà à quoi ont servi les assemblées de Montréal et de Québec, la nomination de L'Espérance comme organisateur en chef et l'attitude nuancée de Patenaude pour faire accepter celui-ci. Même si le problème que présente un pays où sont solidement ancrées des valeurs antagonistes n'est pas résolu, les conservateurs espèrent secrètement qu'un grand succès au Québec aura des effets d'entraînement ailleurs au pays. Pour cela, il faut toutefois résoudre complètement les difficultés d'un parti absent du Québec.

\section{LES DIFFICULTÉS D'UN PARTI FACE À LUI-MÊME - LA DERNIÈRE ÉTAPE}

En préparant le tableau de la réconciliation entre les deux chefs politiques, L'Espérance n'a en effet qu'un but: «to clear the atmosphere ${ }^{47} \gg$. Après plusieurs tergiversations ${ }^{48}$, on convie finalement supporteurs et partisans conservateurs à un rassemblement qui aura lieu au Forum de Montréal, le 4 juin. Quel rôle réserve-t-on alors à Patenaude? L'occasion serait tentante de le présenter aux côtés de son chef. Par la voix de son principal porte-parole dans l'affaire, Joseph Morin ${ }^{49}$, Patenaude fait part aux organisateurs de ses réticences face à cette stratégie. Plutôt que de faire parader Meighen et Patenaude côte à côte sur la même estrade, on s'entend pour une approche plus prudente, ce qui

\footnotetext{
46. L'Événement, «M. Meighen royalement reçu à Bagot», 2 décembre 1925, 7.

47. ANC, Fonds Meighen, L'Espérance à Meighen, 9 mars 1926.

48. ANC, Fonds Meighen, Meighen à Murray Williams, 9 mars 1926; L'Espérance à Meighen, 23 mars 1926; 2 avril 1926; 16 avril 1926; 15 mai 1926; La Patrie, «La manifestation Meighen dans l'est de la ville», 22 mars 1926, 3; «Les conservateurs fêteront M. Meighen en un banquet», 3 avril 1926, 3; «M. Meighen promet de venir le 4», 19 mai 1926, 1; Le Canada, «Un désarroi général dans le camp des conservateurs», 7 juillet 1926, 8; Le Devoir, «Pas de grand banquet à M. Meighen», $1^{\mathrm{er}}$ avril 1926, 1.

49. APA, Fonds Patenaude, Boîte II, Chemise «Campagne - Politique 1926», Morin à Meighen. Cette chemise contient toute la correspondance de Morin concernant l'organisation de l'assemblée du Forum.
} 
n'empêchera pas Patenaude et ses proches de suivre de très près le déroulement de la soirée ${ }^{50}$.

C'est donc en l'absence de Patenaude que Meighen se présente devant une salle bondée ${ }^{51}$. On y entend des discours qui font appel à l'unité du parti et présentent Meighen comme le seul chef politique vraiment capable de défendre les droits des francophones. Ainsi «réchauffée», la salle est prête à entendre le message de son chef. Celui-ci ne déçoit pas son auditoire. Après quelques remarques antilibérales, commentaires partisans de bon aloi énoncés dans sa langue maternelle, Meighen enchaîne dans la langue de Molière et livre des propos qui s'avèrent la pierre d'angle sur laquelle on espère bâtir le succès de la soirée ${ }^{52}$.

Ses paroles au sujet de sa présence au Québec, des relations impériales et de la nécessité de la participation des Canadiens français au bon fonctionnement du gouvernement, réminiscence provenant directement d'idées échangées entre Meighen et Patenaude depuis $1920^{53}$, ont soulevé l'enthousiasme de l'assemblée. Toutefois, la partie du discours qu'il réserve pour parler de Patenaude semble avoir le plus attiré l'attention. Bien qu'on ait voulu l'enrober, l'intervention fait mouche. Le Canada, pourtant hostile aux forces tories, affirme même que «ce fut là le thème le plus saillant de son discours ${ }^{54} \gg$. La phrase tombe à point et, avec des mots choisis ${ }^{55}$, veut mettre un terme à l'aventure indépendante de 1925 . «Tendant un rameau d'olivier, il offre à l'Hon. M. Patenaude un témoignage public de son estime et de son admiration ${ }^{56} »$, soulignent des témoins.

«Quant à l'homme d'État distingué qui conduisit les forces conservatrices de cette province, dit Meighen, je puis l'assurer que je conserve pour lui les sentiments les plus bienveillants et qu'il garde toute mon estime [...]. Je suis convaincu que dans la direction de cette campagne, comme dans l'accomplissement des autres tâches importantes de sa carrière, l'Hon. M. Patenaude s'est laissé diriger par un sentiment très haut de son devoir

50. ANC, Fonds Meighen, Morin à Meighen, 10 juin 1926.

51. Le Devoir, «Nous sommes une nation autonome dans l'Empire britannique et nous entendons le rester», 5 juin 1926, 1; La Patrie, «Près de dix mille personnes écoutent l'Hon. M. Meighen au Forum», 5 juin 1926, 28-33, et «Je crois que les deux races ont besoin de mieux se connaître», 2933.

52. Tel que l'avait prévu L'Espérance, ce geste était important. Voir ANC, Fonds Meighen, L'Espérance à Meighen, 16 avril 1926.

53. Voir Nelson Michaud, «Le nationalisme...», loc. cit.

54. Le Canada, «Quelques questions», 9 juin 1926, 4.

55. ANC, Fonds Meighen, L'Espérance (?) à Meighen, 2 juin 1926.

56. La Patrie, «M. Meighen à Montréal», 7 juin 1926, 4. Le Devoir, «Bloc-notes», 7 juin 1926, 1, reprend la même image du rameau d'olivier. 
envers le pays ${ }^{57} . »$ Un reporter note que «lorsqu'il mentionne le nom de l'Hon. M. Patenaude, des applaudissements partirent de plusieurs côtés de la salle ${ }^{58}$ ». Exagération d'un journaliste sympathique à Patenaude, œuvre bien orchestrée par des meneurs de claque ou réaction spontanée de l'assistance? Peu importe, car l'effet désiré a été obtenu: Meighen vient à la rencontre de Patenaude et les forces conservatrices du Québec acceptent encore ce dernier comme leader. Il ne reste qu'à se donner le baiser de paix.

Patenaude fait toutefois attendre sa réponse. Le succès remporté par l'assemblée du Forum et la cohésion retrouvée par l'équipe conservatrice ${ }^{59}$ auraient pourtant eu de quoi susciter une réaction quasi immédiate de sa part. Toutefois, les jours passent et, malgré la fièvre de l'attente, rien ne paraît. On feuillette fébrilement les quotidiens dans l'espoir d'y trouver une déclaration, mais en vain. Il faut attendre six longues journées avant que ne soit publiée à la une du Devoir, la réaction si anxieusement espérée:

Les déclarations que nous venons d'entendre de la bouche du chef du parti [...] replacent le parti conservateur dans la tradition et donnent raison à notre campagne de l'automne dernier. Nous avons prêché ce que nous croyions être la vraie doctrine conservatrice, et nous sommes bien aise de la retrouver sur les lèvres du chef actuel du parti conservateur. Rappelant le passé et s'inspirant de la pensée des pères de la Confédération, monsieur Meighen recherche à la fois l'unité de son parti et l'unité canadienne. Parlant en mon nom, je désire m'associer à ces sentiments, et j'ai confiance que tous les conservateurs feront de même. Il m'est arrivé de différer d'avis avec mes chefs, et j'ai alors exprimé sans hésitation mais à regret mon dissentiment. Je me sens à l'aise aujourd'hui pour souligner et accueillir favorablement les déclarations de monsieur Meighen ${ }^{60}$.

Cette réponse réjouit évidemment Meighen ${ }^{61}$ et rallie davantage que les meighenistes convaincus. Tour à tour, les Perley ${ }^{62}$, Lockwell ${ }^{63}$ et L'Heureux ${ }^{64}$, comme plusieurs autres patenaudistes inconditionnels, y

\footnotetext{
57. Le Devoir, «Nous sommes une nation autonome...», 5 juin 1926, 1-2.

58. La Patrie, «Je crois que les deux races...», 5 juin 1926, 9. 1926.

59. ANC, Fonds Meighen, Rivard à Meighen, 5 juin 1926; L'Espérance à Meighen, 5 et 7 juin

60. Le Devoir, «M. Patenaude et M. Meighen», 10 juin 1926, 1; Le Canada, «M. Patenaude se rallie à son chef, M. Meighen», 11 juin 1926, 7, reproduit la dépêche «pour mémoire».

61. ANC, Fonds Meighen, Meighen à L'Espérance, 11 juin 1926. Voir aussi APA, Fonds Patenaude, Boîte II, Chemise «Campagne 1926», Meighen à Patenaude, 12 juin 1926.

62. Idem, Perley à Patenaude, 11 juin 1926.

63. Idem, Lockwell à Patenaude, 12 juin 1926.

64. Idem, L'Heureux à Patenaude, 21 juin 1926.
} 
trouvent aussi motif à satisfaction. L'unité du parti semble donc reconquise. Le Devoir résume fort bien la portée du discours de Meighen et son acceptation par Patenaude; analysant la déclaration de ce dernier, un journaliste précise: «On croit qu'elle indique que l'Opposition a renoncé à se chercher un autre chef et que, maintenant, tous les conservateurs du pays, sans exception, ceux de Montréal comme les autres, se tiendront en arrière de M. Meighen. [...] La déclaration de M. Patenaude est donc un triomphe pour la direction du Chef de l'Opposition actuel ${ }^{65}$.»

N'était-ce pas là le but même de l'entreprise? Que Patenaude ait gardé la maîtrise de ses hommes et les ait amenés en même temps que lui à se rallier permet de colmater la large brèche qui existait hier encore au cœur du parti. Cette réconciliation, basée sur une vision commune d'une nation canadienne à solidifier, ce succès stratégique allait prendre une valeur inestimable à la lumière des événements qui sont à la veille de se dérouler en ce mois de juin 1926. Cette réconciliation devait toutefois porter en même temps en elle les affres d'une lutte électorale impitoyable.

\section{LES DIFFICULTÉS D'UN PARTI FACE À UN PARLEMENT AMBIVALENT}

La réconciliation avec Patenaude apportera-t-elle les fruits escomptés, c'est-à-dire la victoire électorale? S'il n'en tient qu'au rôle que Meighen lui réserve, les attentes des organisateurs locaux sont comblées ${ }^{66}$. Patenaude sera la figure de proue conservatrice au Québec: à la suite de la crise politique qui l'amène à devenir Premier ministre, Meighen fait de Patenaude son ministre de la Justice, poste prestigieux. De plus, lors de rencontres qui se multiplient entre le 9 et le 13 juillet ${ }^{67}$, Meighen lui demande conseil, à tel point que Patenaude sera le maître d'œuvre plénipotentiaire ${ }^{68}$ pour combler les postes de ministre réservés à des politiciens du Québec. À la fin d'août, il aura pourvu deux des trois postes vacants: ses choix reposent sur le docteur Eugène Paquet (Santé et Rétablissement civil des soldats), ex-député conservateur-nationaliste de L'Islet, candidat dans Bonaventure, «gros travailleur» du parti. La seconde affectation revient à celui qui tente de conquérir le comté de Bagot pour la troisième fois en dix mois, André Fauteux, qui devient

65. Le Devoir, «On veut se presser de la clore», 14 juin 1926, 1.

66. ANC, Fonds Meighen, Royer à Meighen, 3 juillet 1926.

67. ANC, Fonds Meighen, contient plusieurs lettres et télégrammes qui témoignent de cette activité entourant la formation du nouveau Cabinet; le tout se déroule dans les grands hôtels de Montréal. Voir également, Le Canada, «Une entrevue de M. Meighen avec MM. Patenaude et Fauteux», 9 juillet 1926,8

68. ANC, Fonds Meighen, Meighen à Patenaude, 6 août 1926. 
solliciteur général. Malgré les nombreux noms avancés, on ne peut parvenir à choisir un troisième ministre avant le scrutin et Patenaude doit assumer l'intérim du portefeuille de la Marine et des Pêcheries. On préfère jouer de prudence et éviter toute friction, tout mauvais pas, qui pourraient faire perdre un comté. Chaque geste est longuement évalué, soupesé.

Toutes ces manœuvres produisent-elles l'effet souhaité? Chose certaine, la place que Patenaude occupe réjouit grandement les conservateurs du Québec et la vague d'enthousiasme qui soulève les troupiers bleus de la province suffit à consolider les appuis conquis au moment de la réconciliation Meighen - Patenaude. Ainsi, C. J. Lockwell n'hésite pas à télégraphier au Premier ministre ses sincères félicitations et sa vive satisfaction ${ }^{69}$. Voilà donc un gain appréciable à l'aube d'une campagne électorale. Par ailleurs, les supporters de Rodolphe Monty qui ont fait pression sur Meighen pour que l'on préfère leur homme à Patenaude ${ }^{70}$, entendent, dans l'annonce d'un Cabinet excluant Monty, le glas qui sonne la fin de leurs efforts.

Patenaude a donc la voie libre. D'autant plus que le chef provincial, Arthur Sauvé, qui a fait l'objet d'une attention toute spéciale de la part de L'Espérance depuis le printemps ${ }^{71}$, passe du camp des opposants à la candidature de Patenaude en 1925 à celui de ses plus vifs supporters ${ }^{72}$. Il est vrai que Sauvé n'a plus à perdre son pilier à la législature provinciale, comme c'était le cas dix mois plus tôt. Cependant, l'engagement des députés provinciaux Morin et Saint-Jacques dans les démarches de ralliement à Meighen, constitue une preuve concrète de la nouvelle concorde qui existe entre le Parti conservateur fédéral et le Parti conservateur provincial. Quant à Armand Lavergne, il accepte la candidature dans le comté de Montmagny où il y défend les politiques de son parti ${ }^{73}$. Enfin, l'éditeur du Montreal Daily Star et premier acteur de soutien dans l'épisode de 1925, lord Atholstan, se fait tout miel face au chef

69. Idem, Lockwell à Meighen, 14 juillet 1926. (Télégramme). Plusieurs autres lettres d'organisateurs provenant des quatre coins du Québec apportent des témoignages semblables.

70. ANC, Fonds Meighen. On songe notamment aux lettres d'Herménégilde Boulay, 5 juillet 1926, d'Alfred Mathieu, $1^{\text {er }}$ juillet 1926 et de L.-G. Gravel, 31 juillet 1926 qui, parmi d'autres, se font plus insistantes.

71. Idem, L'Espérance à Meighen, 16, 22, 24 et 26 avril 1926; Meighen à J.-E. Laforce, 5 février 1926.

72. La Patrie, «Avis aux Tories et aux Conservateurs», 6 août 1926, 4 .

73. Idem, «Lavergne est ovationné à Montmagny», 23 août 1926, 14; Le Devoir, «La politique», 27 août, 3 . 
qu'il honnissait moins d'un an plus tôt! Il laisse la campagne de Patenaude se dérouler sans intervenir ${ }^{74}$.

Le triumvirat Patenaude - Meighen - L'Espérance a donc réussi à unifier les troupes. En proclamant à l'unisson le même credo politique, le chef et son lieutenant, indéfectiblement supportés par leur organisateur, ont réussi à relever le défi de l'unité du parti, du moins au Québec. Ils croient alors être prêts à s'engager dans la lutte, appuyés par tous ces groupes qui, hier encore, devaient être considérés comme autant de factions irréconciliables à l'intérieur d'un Parti conservateur fédéral déchiré.

Toutefois, la manière dont Meighen a choisi quelques-uns de ses ministres accélère le dénouement de la crise politique au rythme de laquelle le Canada vit depuis près d'un an. Il faut se souvenir qu'à l'époque, le principe de la responsabilité ministérielle demandait qu'un député appelé au Cabinet démissionne de son siège et que l'électorat de son comté sanctionne en quelque sorte sa nomination ministérielle en le réélisant. Or, dans ce contexte d'un gouvernement minoritaire - un phénomène alors inconnu en Grande-Bretagne et tout aussi impossible au Canada avant l'arrivée en nombre des députés du Parti progressiste en 1921 — Meighen sent un piège pour lequel il n'existe aucun moyen de prévention connu: s'il nomme l'ensemble de son Cabinet à partir de son caucus ou s'il demande à quelques-uns de ses députés de laisser leur siège à des collègues venant de l'extérieur - notamment pour permettre la venue au Cabinet de ministres francophones du Québec - , il se trouve dans l'obligation d'«ouvrir» un nombre suffisant de comtés où autant de candidats libéraux pourraient se faire élire et donner ainsi à leur propre parti une justification numérique, sanctionnée par la volonté populaire, pour revenir au pouvoir.

Meighen contourne cette tradition en nommant des acting ministers privés de leurs émoluments, constituant ainsi un Cabinet qui n'a pas à se présenter devant l'électorat. Les libéraux se saisissent de la question, évoquent la non-légitimité du gouvernement et présentent une motion de blâme à leur endroit. Les progressistes qui avaient causé, par la menace d'un vote réprobateur, le revers de fortune de King, lui sont de nouveau fidèles et, à peine trois jours après être devenu Premier ministre, Arthur

74. Cela fait dire au Montreal Herald, tout comme au Devoir, que le recul pris par Patenaude vis-à-vis de Hugh Graham a causé un froid entre les deux hommes. L'hypothèse n'est pas hors de considération, mais la lettre d'Atholstan à Meighen fait davantage croire à un ralliement du lord. Est-ce là un repli stratégique pour pouvoir mieux contre-attaquer? Chose certaine, malgré ce qu'en disent les journaux libéraux et Le Devoir, Atholstan ne met pas, cette fois, de bâtons dans les roues de l'équipe conservatrice. 
Meighen présente sa démission au gouverneur général qui, cette fois, n'a d'autre choix que de dissoudre le parlement et d'appeler des élections pour tenter de résoudre ce grave problème politique. Meighen n'aura pas su surmonter cette difficulté que présente un Parlement ambivalent, ce qui le force, à toutes fins utiles, à se présenter devant l'électorat, les mains vides, à la tête d'une équipe qui vient d'être défaite en Chambre, laissant du coup l'initiative de la campagne aux libéraux de King.

\section{LES DIFFICULTÉS D'UN PARTI FACE À UN ÉLECTORAT SCEPTIQUE}

Tel que l'avait laissé supposer le rôle qu'on lui avait confié lors de la formation de Cabinet, la place qu'occupe Patenaude au cours de cette campagne électorale en est une bien en vue: dès le lancement, le 20 juillet, à Ottawa, Meighen et Patenaude sont côte à côte, sur la même estrade, prenant tour à tour la parole. On peut désormais considérer Patenaude comme le numéro deux du Parti conservateur au Canada. Pour reprendre les termes de John English, il est le lieutenant de Meighen, comme Ernest Lapointe est celui de Mackenzie King. Et le souhait de plusieurs conservateurs est, qu'advenant une victoire tory au prochain scrutin, il soit possible de faire référence au ministère Meighen-Patenaude, comme on parlait de celui de Macdonald-Cartier.

En termes d'enjeux, les conservateurs doivent d'abord reconquérir une partie importante de l'électorat du Québec, en plus de solidifier leur emprise ailleurs au Canada. Pour ce faire, ils doivent projeter une image de crédibilité et inspirer confiance puisque, notamment au Québec, les troupes conservatrices suscitent plutôt la méfiance. Certes, la présence de Patenaude aux côtés d'un chef qui a lui aussi défendu les principes de l'autonomie canadienne vis-à-vis de l'Empire ou le fait que le nouveau Cabinet comprend le Franco-Ontarien R.-D. Morand - ce qui répond concrètement à une demande de reconnaissance de la dualité canadienne exprimée depuis plus d'un lustre par les nationalistes ${ }^{75}$ — permettent de croire à certains engagements propres à plaire aux électeurs du Québec. Cependant, ces efforts ne semblent pas suffisants.

Tout d'abord, le discours électoral conservateur n'est guère édifiant: Patenaude, Meighen et les conservateurs réclament encore et encore «stabilité des politiques et stabilité politique». En faisant référence à ces concepets, les conservateurs veulent dire qu'eux, et non l'adversaire, sont en mesure d'offrir un programme politique dont la réalisation ne repose pas sur les préférences aléatoires d'un allié circonstanciel, c'est-à-dire les pro-

75. Voir N. Michaud, «Le nationalisme...», loc. cit. 
gressistes — d'où la stabilité des politiques — et ils affirment représenter, en conséquence, la meilleure solution que l'on puisse trouver à la crise que traversent les institutions politiques canadiennes, d'où le retour à la stabilité politique. Patenaude évoque même le principe que c'est par la stabilité politique que passe le salut national ${ }^{76}$. Ces efforts pour amener dans la campagne une dimension programmatique sont sans doute fort louables, mais ils tombent à plat: Patenaude est très mal placé pour louanger les vertus de la stabilité politique, lui qui a fait la navette entre les parlements de Québec et d'Ottawa, lui qui a démissionné en 1917, lui qui a renié Meighen en 1925, pour en être le lieutenant un an plus tard.

En fait, il semble bien que la réconciliation de Patenaude et de Meighen n'aura pas réussi à faire oublier les tergiversations partisanes et l'escapade de 1925. Pis, elle n'aura rien fait avancer au Québec tandis que, selon certains, elle aurait fait perdre des sièges à Meighen en Ontario, éventualité pressentie par le Toronto Star ${ }^{77}$ et réitérée plus tard par un organisateur de cette province qui confie à Meighen que: «Mr. Patenaude is anything but a hindrance to the Conservative Party ${ }^{78}$.»

En réalité, les conservateurs perdent dix sièges dans la province voisine. Toutefois, malgré les apparences, il semble bien que «the Ontario ridings likely to be most annoyed by the speech indicate that he had lost little there ${ }^{79} \gg$, comme le précise James Murray Beck. Cette nuance est confirmée par Meighen pour qui ce n'est pas tant le contenu du discours d'Hamilton (qui a été à la base de cette réconciliation) qui pose problème au Canada anglais, que le fait que, devant les protestations que ses propos ont suscitées, Meighen a accepté de placer la lampe sous le boisseau et de taire sa position. Plus de trente ans après ces événements, Meighen confiera d'ailleurs à l'historien Roger Graham, qu'à son retour de Bagot où il avait repris ce thème, certains conseillers avaient fait pression sur lui:

in the strongest terms to let the subject drop, or, rather, let it lie quiet until immediate antipathies would cool down. To these importunities, I, unfortunately, acceded. This, I frankly confess, was a mistake in tactics. I should have unhesitatingly defended my position and [...] arranged for immediate public speeches in Canada [...] and proclaimed the lines of the Hamilton speech as definitely my policy, and made it the policy of the Conservative party. To my mind,

76. La Patrie, «L'Hon. M. Meighen ouvre sa campagne dans la province de Québec», 2 août $1926,1-2$

77. Toronto Star, 13 juin 1926, cité dans Le Devoir, «Bloc-Notes», 14 juin 1926, 1.

78. ANC, Fonds Meighen, P.-C. Locke à Meighen, 15 septembre 1926.

79. James Murray Beck, op. cit., 179. 
this was the only major, or at any rate the most important, mistake I made in public life. There would even so probably have been some who would have persevered in their antagonism, but they would have been very, very few, and there might have been not at all ${ }^{80}$.

Si l'évaluation du chef conservateur peut, finalement, sembler optimiste, il n'en demeure pas moins qu'elle révèle qu'à l'extérieur du Québec, le Parti conservateur fait aussi face à un grave problème de crédibilité dont la source est un message ambigu.

Les libéraux, quant à eux, exploitent cette faiblesse des conservateurs. King ne paraît pratiquement pas au Québec et il laisse aux Ernest Lapointe, Lucien Cannon et P.-J.-A. Cardin le soin de mener une campagne au tambour des attaques ad hominem et de la dénonciation d'une supposée persécution du Québec par les tories impérialistes. Les libéraux placent ainsi constamment leurs adversaires sur la défensive, les forçant à consacrer toutes leurs énergies à reconstruire leur image. Ils ne leur laissent pratiquement aucune marge de manœuvre qui pourrait leur permettre d'aborder leurs propres priorités au cours de la campagne. Une analyse des propos tenus par les libéraux au cours de la campagne permet aussi de constater qu'ils ont littéralement dégonflé les voiles qui devaient porter l'équipe Meighen-Patenaude: alors que ces derniers avaient décidé de jouer la carte de l'autonomie canadienne tout au long de leur démarche de réconciliation, précisément en vue de s'attacher le Québec, c'est King et ses hommes qui, durant toute la campagne, lancent des appels à l'autonomie canadienne en brandissant le «scandale constitutionnel» et le «retour à l'état de colonie» que représente l'attitude du gouverneur général ${ }^{81}$ qui a refusé la dissolution du Parlement au chef libéral, pour ensuite l'accorder à Meighen. Qui plus est, même le scandale des douanes, soit le sujet le plus dommageable que les conservateurs pourraient exploiter afin de miner la crédibilité des libéraux, est récupéré par l'équipe libérale ellemême: dès le début de la campagne, La Presse rapporte les propos de Cardin qui affirme: «Vous verrez toute la boue que l'on amoncellera contre notre province, contre les Canadiens français. L'enquête sur les douanes n'a eu pour objet que de déclencher une attaque contre Québec seulement ${ }^{82} . »$

80. ANC, Fonds Meighen, «Mémoire à Roger Graham», s.d., 42.

81. Cette accusation avait d'autant plus d'emprise qu'à l'époque, non seulement le gouverneur général avait-il le statut constitutionnel de représentant de la Couronne britannique, mais il s'agissait d'un ressortissant de la Grande-Bretagne que le roi dépêchait à titre d'émissaire dans son Dominion, poursuivant ainsi la tradition coloniale.

82. La Presse, 21 juillet 1926, 1. 
Patenaude et l'équipe conservatrice sont déstabilisés. Le passif qui grève leur position donne d'autant plus de mordant aux attaques des libéraux. Plutôt que d'imposer leurs idées, les «bleus» doivent d'abord tenter de rétablir les faits, demeurant ainsi à la remorque de la cadence marquée par leurs adversaires. Certes, tout au long de la campagne, les thèmes purement nationaux sont parfois présents, mais ils sont ensevelis sous la lourde chape de la harangue partisane, un jeu auquel les conservateurs partent perdants.

Cela n'empêche pas les quotidiens de procéder à des analyses conformes à leurs allégeances respectives, les uns référant à une réponse chaleureuse de l'électorat vis-à-vis des propositions conservatrices, les autres évoquant un accueil des plus froids. Les témoignages externes, eux, laissent malgré tout entrevoir que l'on réserve, au moins en début de campagne, un accueil sympathique au chef tory et à ses troupes ${ }^{83}$. Attestant de la campagne au Québec, on mentionne même que "plus d'un habitant, rencontrant Meighen, est surpris de voir un homme intelligent, apparemment bien disposé, au lieu du monstre féroce qu'il imaginait ${ }^{84} \gg$. Pourtant, selon ce que note Le Devoir, «les apparences sont moins brillantes que l'an dernier dans la province de Québec ${ }^{85}$ ».

Effectivement, les jours semblent moins radieux pour les conservateurs. Pour Meighen, «Quebec is an enigma ${ }^{86} . »$ Patenaude n'a-t-il pas sa part de problèmes? Les libéraux font grand bruit du fait qu'il prenne beaucoup de temps à désigner le comté où il se présentera, contraste une fois de plus marqué avec les propos du politicien qui parle continuellement de stabilité ${ }^{87}$. Au début d'août, lorsque Patenaude opte finalement pour un retour dans Jacques-Cartier, Le Soleil annonce que «le caméléon qui rampait sur la carte de notre province depuis quelques semaines à la recherche d'un comté où il pourrait se faire battre, a enfin pris une décision ${ }^{88}$ ».

Une anticipation établie d'un point de vue stratégique pourrait laisser croire que les conservateurs rassembleront leur énergie et profiteront des dernières semaines de la campagne pour passer à l'attaque et récupérer les votes nécessaires à la conquête du pouvoir. Chose qui peut sembler surprenante, on les voit plutôt prêts à lancer la serviette: la côte à gravir leur

83. Roger Graham, Arthur Meighen. A Biography, and Fortune Fled (Toronto, Clarke-Irwin, 1962), 453-460.

84. Robert Rumilly, Henri Bourassa. La vie publique d'un grand Canadien (Montréal, Éditions de l'Homme, 1953), 685.

85. Le Devoir, «La politique», 3 août 1926, 3

86. ANC, Fonds Meighen, Meighen à Frank M. Field, 14 juillet 1926.

87. Le Canada, «Professeur en stabilité», 30 août 1926, 4.

88. Le Soleil, «Le caméléon se case», 9 août 1926, 12. 
parait vraisemblablement trop longue ou trop abrupte et ils sentent que le temps leur manque. Même La Patrie diminue de jour en jour sa couverture de la campagne conservatrice et, sur le terrain, les dernières assemblées ne livrent que redites et radotages. À l'occasion de certaines de ces réunions, les conservateurs mutilent des photos de King pendant que les libéraux bombardent la tribune des orateurs bleus de tomates trop mûres et d'œufs trop vieux ${ }^{89}$. Deux jours avant le scrutin, il arrive même à Patenaude de se présenter devant une foule qui ne lui laisse pas prononcer un mot d'un discours que l'on a déjà trop entendu ${ }^{90}$.

En fait, malgré l'importance que lui reconnaît Richard Jones ${ }^{91}$, la campagne électorale fut des plus ternes. Le ton des discours et des débats tout comme la publicité contenue dans les différents journaux en témoignent abondamment. Alors qu'en 1925, on s'ingéniait à trouver des slogans, des scénarios, des images qui frappent, la propagande électorale de la campagne de 1926 ne repique que quelques thèmes exploités dans les discours qui étaient eux-mêmes souvent dépourvus de grandes idées. La campagne a mis aux prises des adversaires aux ressources humaines épuisées, aux caisses électorales vidées et a finalement profité à ceux qui ont opté pour la tactique la moins complexe.

Au soir du 14 septembre, King remporte 119 sièges. Avec l'appui des tierces formations de l'opposition, il obtient la majorité des voix au Parlement. Les conservateurs n'ont plus que 91 représentants aux Communes, Arthur Meighen subissant lui-même la défaite dans Portage-laPrairie. Au Québec, la situation reste inchangée: les quatre députés conservateurs anglophones sont réélus et Patenaude, battu de nouveau. Quant à Bourassa, il est de retour à Ottawa avec ses soixante amis libéraux. Le pourcentage du vote obtenu par les bleus est satisfaisant mais, comme l'a fait remarquer James Murray Beck, «they spread their votes so evenly that they again failed to elect a single French-Canadian ${ }^{92} \gg$. Patenaude, qui a suivi le dépouillement du scrutin grâce à un appareil télégraphique installé dans la maison d'un ami à Lachine, «apprit sa défaite avec calme. Il semblait plus préoccupé du sort de son parti que du $\operatorname{sien}^{93} . »$ Le lieutenant se retire sans plus de commentaires et passe plusieurs jours, seul, à Laval-

89. Le Devoir, «À Lachine, hier soir», 14 septembre 1926, 7, et La Patrie, «L'Hon. M. Patenaude a parlé hier soir à Lachine, malgré une bande de crieurs à gages», 14 septembre 1926, 5. $1926,7$.

90. La Patrie, «Des crieurs à gages veulent étouffer la voix de M. Patenaude», 13 septembre

91. Richard Jones, Vers une hégémonie libérale. Aperçu de la politique canadienne de Laurier à King (Sainte-Foy, La librairie des Presses de l'Université Laval, 1980), 203.

92. James Murray Beck, op. cit., 187.

93. La Patrie, «Ce qui s'est passé dans le district de Montréal, hier», 15 septembre 1926, 2. 
sur-le-Lac ${ }^{94}$. Bien qu'il participera à quelques activités ultérieures du parti, il n'est plus question pour lui d'autres responsabilités partisanes officielles.

En fait, si l'on considère l'analyse succincte et précise que John Herd Thompson et Allan Seager ${ }^{95}$ font des résultats de l'élection, nous nous apercevons que la facilité avec laquelle les libéraux ont pu aller chercher des votes progressistes n'a d'égale que l'inhabileté des conservateurs à se faire accepter au Québec. Les conservateurs n'auront donc pas réussi à convaincre un électorat sceptique à leur égard.

\section{LE BILAN}

En prenant comme fil conducteur une partie de la carrière politique d'Ésioff-Léon Patenaude, il nous a été possible de pénétrer dans les coulisses d'un épisode de la vie politique canadienne de l'entre-deux-guerres. Nous y avons analysé comment le Parti conservateur fédéral a tenté de reprendre le rang de parti national auquel il était habitué, lui qui avait réussi cette confédération des colonies qui allait devenir le Canada moderne, mais qui, lors de l'élection de 1921, la première conduite par Arthur Meighen, avait glissé au troisième rang. Un laborieux travail de reconstruction devait alors commencer.

Avec le recul, on pourrait dire que Meighen et Patenaude ont accompli assez pour qu'on leur crédite un travail d'envergure, quoiqu'insuffisant: après tout, sous l'égide de Patenaude, le Parti conservateur a réussi à presque doubler l'appui recueilli auprès des électeurs du Québec, passant de 18,4\% à 34,3\% des votes exprimés entre 1921 et 1926. Patenaude a ainsi contribué à redonner une assise viable à son parti. Cependant, ces efforts ne furent pas suffisants pour mener à la victoire; en politique comme ailleurs, on ne se souvient guère de ceux et celles qui se classent au deuxième rang et ce, malgré les énergies investies. Le véritable défi, celui de la conquête du pouvoir, n'aura donc pas été relevé.

Parmi les causes de cet échec, il faut compter l'incapacité du parti à surmonter efficacement des difficultés dans divers domaines. D'une part, l'unité du parti a été refaite avec un certain succès, notamment au Québec; mais les incertitudes que ce succès a générées n'ont pas été efficacement gérées, ce qui devait constituer un boulet qui allait entraver la marche des politiciens vers d'autres réussites. En fait, plus que la

94. Madame Rose Prévost (fille d'É.-L. Patenaude), Entrevue avec l'auteur.

95. John Herd Thompson et Allan Seager, op. cit., 124-128. 
difficulté d'harmoniser une position politique acceptable à l'ensemble d'un pays divisé, l'incapacité de résoudre les ambiguittés associées aux changements de cap qu'ont effectués autant Patenaude, de retour au sein du parti national, que Meighen, qui tempère sa position vis-à-vis de l'Empire, aura coûté cher. D'autre part, coincés dans une camisole de force constitutionnelle, les conservateurs n'ont pas réussi à gagner la confiance d'un Parlement ambivalent, pas plus que celle d'un électorat sceptique qui, à un Meighen aux positions en apparence hésitantes et mal définies, a préféré un leader sûr de lui et prêt à tout pour conserver le fauteuil de Premier ministre du pays.

En prenant comme point d'ancrage la carrière politique de Patenaude, nous pouvons nous interroger sur les choix du chef tory en vue de rebâtir son parti. En se rapprochant de Patenaude, Meighen s'est-il trompé d'homme? En accueillant à bras ouverts son lieutenant tel l'enfant prodigue, en reniant le fils fidèle qu'était Monty, Meighen a-t-il commis l'erreur politique qui lui a coûté la direction du pays? Il faut aussi considérer qu'en 1926, Patenaude est, lui aussi, un homme public dont l'adversaire libéral a miné la carapace et altéré la crédibilité politique. De plus, les assises de son ralliement sont vite balayées par l'adversaire: le nationalisme canadien est, au mieux, récupéré par les libéraux et, au pis, il cède le pas à la lutte électorale partisane pure et ne représente plus, lors de la campagne électorale, l'enjeu autour duquel on avait, jusqu'à ce jour et depuis plus de quinze ans, réuni les partisans conservateurs du Québec francophone. Patenaude, son chef et son parti en ont payé la note.

L'analyse de cet épisode de la vie politique du Québec et du Canada répond à certains éléments d'interprétation qui étaient, jusqu'à maintenant, demeurés obscurs. En apportant ce nouvel éclairage, nous avons toutefois le sentiment qu'il suscite autant de questions qu'il n'apporte de réponses. L'une des avenues intéressantes qui s'ouvre devant nous repose sur la similarité de la situation politique de la fin des premier et dernier quarts du XX ${ }^{\mathrm{e}}$ siècle: insatisfaction du Québec, émergence des régionalismes, déroute du Parti conservateur et emprise libérale sur un pouvoir que l'on veut conserver à tout prix, pour ne donner que quelques exemples. Il serait aussi intéressant de reprendre l'analyse à l'aide de modèles empruntés à la science politique proprement dite, que ce soit, par exemple, celui de la structuration des pouvoirs de Lemieux ${ }^{96}$ ou l'un

96. Voir Vincent Lemieux, La structuration du pouvoir dans les systèmes politiques (SainteFoy, Les Presses de l'Université Laval, 1989) et, du même auteur, Éléments d'une théorie politique des voix (Sainte-Foy, Laboratoire d'études politiques (Université Laval), 1995). 
de ceux proposés par Garrigou ${ }^{97}$, afin de déterminer la part des influences structurelles et conjoncturelles dans l'élaboration et l'acceptation par l'électorat, des positions défendues par les conservateurs. Nous trouvons là matière à autant de questions nouvelles et stimulantes que notre analyse pourrait susciter, mais dont les réponses appartiennent à ceux et celles qui voudront adopter une approche différente et y consacrer des heures de recherche que nous savons d'avance passionnantes.

97. Alain Garrigou, «Conjoncture politique et vote», dans Daniel Gaxie, dir., Explication $d u$ vote. Un bilan des études électorales en France (Paris, Fondation nationale des sciences politiques, 1985. 